\title{
Inhibition enhances capacity of sequence replay: a mean field model
}

\author{
Álvaro Tejero-Cantero ${ }^{1,2^{*}}$, Axel Kammerer ${ }^{1,2}$, Christian Leibold ${ }^{2}$ \\ From Twentieth Annual Computational Neuroscience Meeting: CNS*2011 \\ Stockholm, Sweden. 23-28 July 2011
}

Sequences of neuronal activity patterns can be stored in networks of binary neurons with binary synapses. We investigate different forms of inhibition and their effect on such sequence memory, extending on a mean field approach [1]. There it was shown that successful replay requires a minimum degree of coding sparseness and that the capacity of the network increases as the code becomes sparser (Fig. 1). Here, we find that the introduction of global inhibition feedback makes sequence replay possible with an even sparser code, thereby increasing the memory capacity of the network (Fig. 1). At the same time, the range of firing thresholds compatible with replay becomes broader, suggesting a more robust behavior with noisy, biological neurons.

We further analyzed the effect of nonoptimal replay conditions: The replay performance degrades gracefully with the network exhibiting transient memory replay before falling into a state of silence or non memoryrelated activity. The regions of stable replay calculated from the mean field model were verified in cellular simulations.

\section{Author details}

${ }^{1}$ Graduate School of Systemic Neurosciences, 82152 Martinsried, Germany. Division of Neurobiology, Department of Biology II, LMU Munich, 82152 Martinsried, Germany.

Published: 18 July 2011

\section{Reference}

1. Leibold C, Kempter R: Memory capacity for sequences in a recurrent network with biological constraints. Neural Computation 2006, 18:904-941.

\section{doi:10.1186/1471-2202-12-S1-P195}

Cite this article as: Tejero-Cantero et al: Inhibition enhances capacity of sequence replay: a mean field model. BMC Neuroscience 2011 12(Suppl 1):P195.

\footnotetext{
* Correspondence: tejero@bio.Imu.de

${ }^{1}$ Graduate School of Systemic Neurosciences, 82152 Martinsried, Germany
} Full list of author information is available at the end of the article

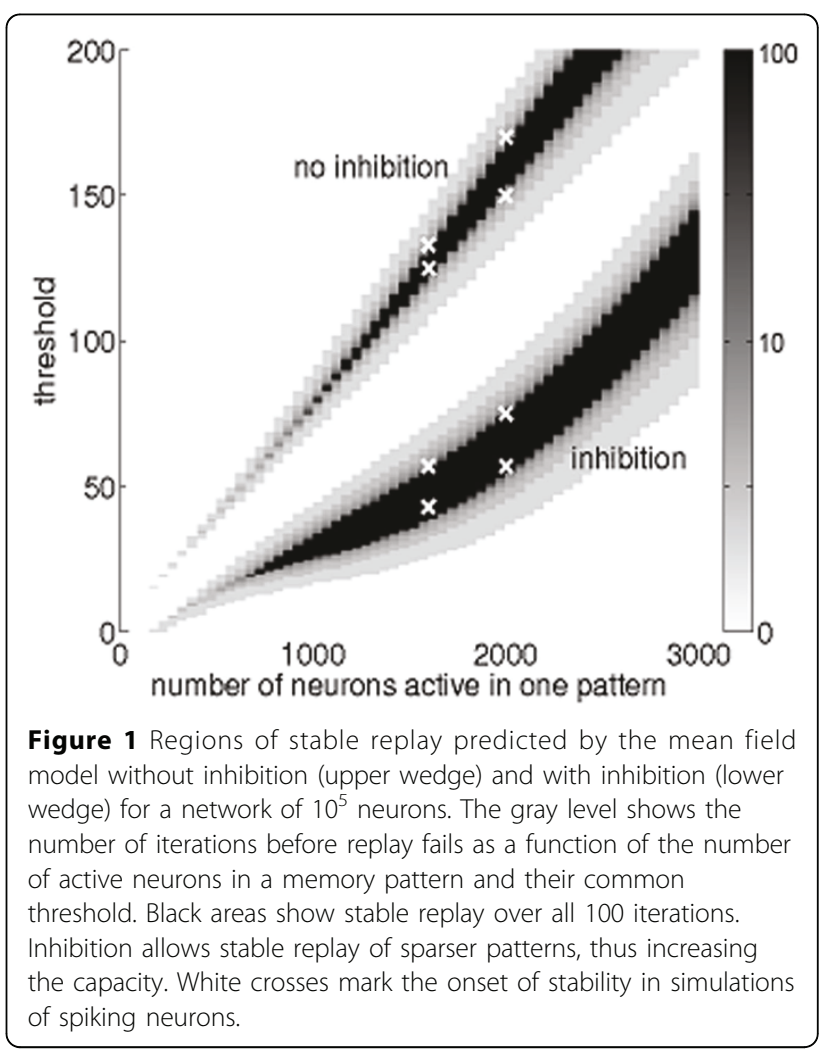

